\title{
On Soft totally bounded sets
}

\section{B.Surendranath Reddy ${ }^{1}$ and Sayed Jalil ${ }^{2}$}

\author{
${ }^{1}$ Assistant Professor, School of Mathematical Sciences, \\ Swami Ramanand Tirth Marathwada University, Nanded, India \\ ${ }^{2}$ Research Scholar, School of Mathematical Sciences, \\ Swami Ramanand Tirth Marathwada University, Nanded, India
}

Keywords: Soft set, Soft point, Soft metric, Soft open ball, Soft bounded set.

\begin{abstract}
In this paper, we investigate some result on soft bounded and soft totally bounded sets and derive relation among them. We investigate some necessary conditions on soft totally bounded sets. We also prove soft totally bounded set is separable.
\end{abstract}

\section{Introduction}

Many times real life problems involve uncertainties, imprecision and vagueness. In particular, such classes of problems arise in economics, engineering environmental sciences, medical sciences, etc. In course of time, a number of mathematical theories such as probability theory, fuzzy set theory, set theory, interval mathematics theory, vague set theory, etc. formulated to solve such problems, have been found only partially successful. The major reason for the difficulties arising with the above mentioned theories is due to the inadequacies of their parameterization tools ([3]).In order to overcome these difficulties, Molodtsov [3] introduced soft set theory as a completely new mathematical tool. This prototype work of Molodtsov, besides, included directions for further research, especially discovering new operations on soft sets and their properties. In recent year many researches like Feng et al.([1]), Maji et al.([2]) years. shabir and Naz ([5]), sujao Das and Samanta ([6],[7]), sadi Bayrramov and Cigdem Gunduz ([4]), have contributed to the development sof set theory. In this paper, section 1 is about preliminaries. In section 2, we derive some results on soft bounded sets in terms of soft open balls and diameter. In section 3, we investigate soft totally bounded sets with examples and prove some necessary conditions of soft totally bounded sets.

\section{Preliminaries}

In this section we recall some basic definitions and results about soft sets.

Definition 1.1[3] Let $U$ be the universe and E be the set of parameters. Let $P(U)$ denotes the power set of $U$ and A be a non empty subset of $E$. A pair $(F, A)$ is called a soft set over $U$ where $F$ is given by $F: A \rightarrow P(U)$.

In other words, the soft set is a parameterized family of subsets of the set $U$. For $\varepsilon \in A, F(\varepsilon)$ may be considered as the set of $\varepsilon$-elements of the set $(F, A)$ or as the set of $\varepsilon$-approximate elements of the soft set. i.e. $(F, A)$ is given as consisting of collection of approximation: $(F, A)=\{F(\varepsilon) / \varepsilon \in A\}$.

In other words, the soft set is a parameterized family of subsets of the set $U$. For $\varepsilon \in A, F(\varepsilon)$ may be considered as the set of $\varepsilon$-elements of the set $(F, A)$ or as the set of $\varepsilon$-approximate elements of the soft set. i.e. $(F, A)$ is given as consisting of collection of approximation:

$(F, A)=\{F(\varepsilon) / \varepsilon \in A\}$.

Definition 1.2[1] For two soft sets $(F, A)$ and $(G, B)$ over a common universe $U$, we say that $(F, A)$ is soft subset of $(G, B)$ if 
1. $A \subseteq B$ and

2. $\forall e \in A, F(e) \subseteq G(e)$.

and it is denoted by $(F, A) \subseteq(G, B)$.

Definition 1.3[2] Two soft sets $(F, A)$ and $(G, B)$ over a common universe $U$ are said to be equal if $(F, A)$ is a soft subset of $(G, B)$ and $(G, B)$ is a soft subset of $(F, A)$.

Definition 1.4[2] The union of two soft sets $(F, A)$ and $(G, B)$ over the common universe $U$ is the soft set $(H, C)$, where $C=A \cup B$ and $\forall e \in C$,

$$
H(e)=\left\{\begin{array}{cl}
F(e), & \text { ife } \in A \backslash B ; \\
G(e), & \text { ife } \in B \backslash A: \\
F(e) \cup G(e), & \text { ife } \in A \cap B .
\end{array}\right.
$$

and is denoted by $(F, A) \tilde{U}(G, B)=(H, C)$.

Definition 1.5[2] The intersection of two soft sets $(F, A)$ and $(G, B)$ over the common universe $U$ is the soft set $(H, C)$ where $C=A \cap B$ and $\forall e \in C, H(e)=F(e) \cap G(e)$ and is denoted by $H(C, A)=F(e) \widetilde{\cap} G(e)$.

Definition 1.5[2] The intersection of two soft sets $(F, A)$ and $(G, B)$ over the common universe $U$ is the soft set $(H, C)$ where $C=A \cap B$ and $\forall e \in C, H(e)=F(e) \cap G(e)$ and is denoted by

$$
H(C, A)=F(e) \widetilde{\cap} G(e) .
$$

Definition 1.6[5] The difference $(H, E)$ of two soft sets $(F, E)$ and $(G, E)$ over $U$ is denoted by $(F, E) \backslash(G, E)$ and is defined as $H(e)=F(e) \backslash G(e), \forall e \in E$.

Definition 1.7[1] The complement of a soft set $(F, A)$ over $U$ is denoted by $(F, A)^{c}$ and is defined as $(F, A)^{c}=\left(F^{c}, A\right)$, where $F^{c}: A \rightarrow P(U)$ is a mapping given by $F^{c}(\lambda)=U-F(\lambda)^{c}, \forall \lambda \in A$ i.e. $(F, A)^{c}=\left\{F\left(e_{i}\right)^{c}, \forall, e_{i} \in A\right\}$.

Definition 1.8[2] A soft set $(F, E)$ over $U$ is said to be a null soft set denoted by $\widetilde{\emptyset}_{\text {if }}$ $F(e)=\emptyset, \forall e \in E$.

Definition 1.9[2] A soft set $(F, E)$ over $U$ is said to be a absolute soft set denoted by $\widetilde{U}$ if $F(e)=U, \forall e \in E$.

Definition 1.10[7] Let $X$ be a non empty set and $E$ be a non empty parameter set then the function $\varepsilon: E \rightarrow X$ is said to be soft element of $X$

A soft element $\mathcal{\varepsilon}$ is said to belongs to a soft set $(F, A)$ of $X$ if $X$ if $\varepsilon(e) \in A(e), \forall e \in A$ and is denoted by $\varepsilon \widetilde{\in}(F, A)$. 
Definition 1.11[7] Let $\mathbb{R}$ be the set of real numbers and $\mathfrak{B}(\mathbb{R})$ the collection of all non empty bounded subsets of $\mathbb{R}$ and $\mathrm{A}$ be a set of parameters then the mapping $F: A \rightarrow \mathfrak{B}(\mathbb{R})$ is called a soft real set. It is denoted by $(F, A)$. In particular, if $(F, A)$ is singleton soft set then identifying $(F, A)$ with the corresponding soft element, it will be called a soft real number. We denote soft real numbers by $\tilde{r}, \tilde{s}, \tilde{t}$ and $\tilde{r}, \tilde{s}, \tilde{t}$ will denote a particular type of soft numbers such that $\tilde{r}(\lambda)=r, \forall \lambda \in A$ etc.

Definition 1.12[6] A soft set $(P, A)$ over $X$ is said to be a soft point if there is exactly one $\lambda \in A$, such that $P(\lambda)=x$, for some $x \in X$ and $P(\mu)=\emptyset, \forall \mu \in A\{x\}$.

It is denoted by $P_{\lambda}{ }^{x}$.

Definition 1.13[6] A soft point $P_{\lambda}{ }^{x}$ is said to belong to a soft set $(F, A)$ if $\lambda \in A$ and $P(\lambda)=\{x\} \subset F(\lambda)$ and we write $P_{\lambda}^{x} \widetilde{\in}(F, A)$.

Dentition 1.14[6] Two soft points $P_{\lambda}{ }^{x}$ and $P_{\mu}{ }^{y}$ are said to be equal if $\lambda=\mu$ and $P(\lambda)=P(\mu)$ i.e. $x=y$. Thus $P_{\lambda}^{x} \neq P_{\mu}^{y} \Leftrightarrow x \neq y$ or $\lambda \neq \mu$.

Theorem 1.15[6] The union of any collection of soft points can be considered as a soft set and every soft set can be expressed as union of all soft points belonging to it, i.e $(F, A)=\bigcup_{p_{\lambda}{ }^{x} \widetilde{E}(F, A)} P_{\lambda}^{x}$.

Let $X$ be an initial universal set and $A$ be a non empty set of parameters. Let $\tilde{X}_{\text {be the absolute soft }}$ set. Let Let $S P(\tilde{X}) \mid 0$ be the collection of all soft points of $\tilde{X}$. Let $\mathbb{R}\left(A^{*}\right)$ denote the set of all non negative soft real numbers.

Definition 1.16[6] A mapping $\tilde{d}: S P(\tilde{X}) \times S P(\tilde{X}) \rightarrow \mathbb{R}(A)$ is said to be a soft metric on the soft set $\tilde{X}$ if

$$
\begin{aligned}
& \text { i) } \tilde{d}\left(P_{\lambda}{ }^{x}, P_{\mu}{ }^{y}\right) \cong \tilde{0}, \quad \forall P_{\lambda}{ }^{x}, P_{\mu}{ }^{y} \tilde{\in} \tilde{X}, \\
& \text { ii) } \tilde{d}\left(P_{\lambda}{ }^{x}, P_{\mu}{ }^{y}\right)=\tilde{0}, \quad \text { if and only if } P_{\lambda}{ }^{x}=P_{\mu}{ }^{y}, \\
& \text { iii) } \tilde{d}\left(P_{\lambda}{ }^{x}, P_{\mu}{ }^{y}\right)=\tilde{d}\left(P_{\mu}{ }^{y}, P_{\lambda}{ }^{x}\right), \forall P_{\lambda}{ }^{x}, P_{\mu}{ }^{y} \tilde{\in} \tilde{X}, \\
& \text { iv) } \tilde{d}\left(P_{\lambda}{ }^{x}, P_{\mu}{ }^{y}\right) \cong \tilde{d}\left(P_{\lambda}{ }^{x}, P_{\gamma}{ }^{z}\right)+\tilde{d}\left({P_{\gamma}}^{z}, P_{\mu}{ }^{y}\right), \forall P_{\lambda}{ }^{x}, P_{\mu}{ }^{y} \tilde{\in} \tilde{X} .
\end{aligned}
$$

The soft set $\tilde{X}_{\text {with the soft metric }} \tilde{d}$ on $\tilde{X}$ : is called a soft metric space and is denoted by $(\tilde{X}, \tilde{d} E)$ or $(\tilde{X}, \tilde{d})$.

Example 1.17 Let $X$ be a non empty set and $E$ be a non empty set of parameters. Let $\tilde{X}_{\text {be }}$ the absolute soft set i.e $F(\lambda)=X, \forall \lambda \in A$, where $(F, A)=\widetilde{X}$. 
Define $\tilde{d}: S P(\tilde{X}) \times S P(\tilde{X})) \rightarrow \mathbb{R}(A)$ by, $\tilde{d}\left(P_{\lambda}^{x}, P_{\mu}^{y}\right)=\left\{\begin{array}{l}\tilde{0}, \text { if } P_{\lambda}^{x}=P_{\mu}{ }^{y} \\ \tilde{1}, \text { if } P_{\lambda}{ }^{x} \neq P_{\mu}{ }^{y}\end{array}\right.$

Then $\tilde{d}$ is a soft metric and is called soft discrete metric and $(\tilde{X}, \tilde{d} E)$ is called soft discrete metric space.

Definition 1.18[6] Let $(\tilde{X}, \tilde{d} E)$ be a soft metric space and $\tilde{r}$ be a non negative soft real number. Then the set $B\left(P_{\lambda}{ }^{x}, \tilde{r}\right)=\left\{P_{\mu}^{y} \tilde{\in} \tilde{X} \mid \tilde{d}\left(P_{\lambda}{ }^{x}, P_{\mu}{ }^{y}\right) \widetilde{r}\right\}$ is called a soft open ball with center $P_{\lambda}^{x}$ and of radius $\tilde{r}$.

Definition1.19[6] Let $(\tilde{X}, \tilde{d} E)$ be soft metric space $(Y, A)$ be a non null soft subset of $\tilde{X}$ then the diameter of $(Y, A)$ is denoted by $\operatorname{diam}((Y, A))$ and for any $\gamma \in A$, it is defined as, $\operatorname{diam}((Y, A))(\gamma)=\sup \left\{\tilde{d}\left(P_{\lambda}{ }^{x}, P_{\mu}^{y}\right)(\gamma) \mid P_{\lambda}{ }^{x}, P_{\mu}{ }^{y} \tilde{\in}(Y, A)\right\}$.

Example 1.20 Let $(\tilde{X}, \tilde{d} E)$ be soft discrete metric space and let $(Y, A)$ be a non null soft subset of $\tilde{X}$, then $\operatorname{diam}((Y ; A))=\tilde{1}$.

Theorem 1.21[6] Let $(\tilde{X}, \tilde{d} E)$ be soft metric space then i) $\operatorname{diam}((Y, A))=\tilde{0} \Leftrightarrow(Y, A)$ consists of single soft element.

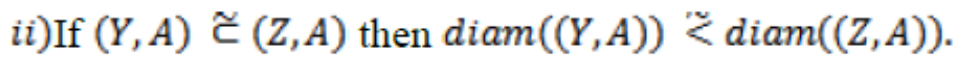

Definition 1.22[6] A soft metric space $(\tilde{X}, \tilde{d} E)$ is said to be soft separable if there exists a countable soft subset $(Y, A)$ of $\tilde{X}$ such that $(Y, A)$ is dense in $\tilde{X}$.

\section{Soft Bounded sets}

In this section we discuss soft bounded sets and prove some of their properties.

Definition 2.1 Let $(\tilde{X}, \tilde{d} E)$ be a soft metric space and $(Y, A)$ be a non null soft subset of $\tilde{X}$. Then we say that $(Y, A)$ is soft bounded if there exists $P_{\lambda}^{x} \tilde{\in} \tilde{X}$ and a soft real number $\tilde{\varepsilon}>0$ such that $(Y, A) \simeq B\left(P_{\lambda}^{x}, \tilde{\varepsilon}\right)$.

Theorem 2.2 Let $(\tilde{X}, \tilde{d} E)$ be soft metric space and $(Y, A)$ be non empty soft subset of $\tilde{X}$ then $(Y, A)$ is soft bounded if and only if the diameter of $(Y, A)$ is finite. 
Proof: Suppose $(Y, A)$ is soft bounded. Then there exist $P_{\lambda}^{x} \tilde{\in} \tilde{X}$ and $\tilde{\varepsilon} \widetilde{>0}$ such that $(Y, A) \simeq B\left(P_{\lambda}^{x}, \tilde{\varepsilon}\right)$.

Then $\tilde{d}\left(P_{\lambda}{ }^{x}, P_{\mu}^{y}\right) \approx \tilde{\varepsilon}, \forall P_{\lambda}{ }^{x} \widetilde{\in}(Y, A)$,

By triangle inequality, for any $P_{\mu}{ }^{y}, P_{\gamma}{ }^{z} \widetilde{\in}(Y, A)$,

$\tilde{d}\left(P_{\lambda}{ }^{x}, P_{\mu}{ }^{y}\right) \widetilde{\leq} \tilde{d}\left(P_{\lambda}{ }^{x}, P_{\gamma}{ }^{z}\right)+\tilde{d}\left(P_{\gamma}{ }^{z}, P_{\mu}{ }^{y}\right) \approx \tilde{2} \tilde{\varepsilon}$

$\Rightarrow \operatorname{Sup}\left\{\tilde{d}\left(P_{\lambda}{ }^{x}, P_{\mu}{ }^{y}\right): P_{\lambda}{ }^{x}, P_{\mu}{ }^{y} \tilde{\in}(Y, A)\right\} \approx \tilde{2} \tilde{\varepsilon}$

$\Rightarrow \operatorname{diam}((Y, A)) \approx \tilde{2} \tilde{\varepsilon}$

Therefore the diameter of

$(Y, A)$ is finite.

Conversely suppose assume that the diameter of $(Y, A)$

Let $\operatorname{diam}((Y, A))=\tilde{l}$, for some $\tilde{l} \sim \tilde{0}$

$\Rightarrow \tilde{d}\left(P_{\lambda}{ }^{x}, P_{\mu}{ }^{y}\right) \widetilde{l} \tilde{l}, \forall P_{\lambda}{ }^{x}, P_{\mu}{ }^{y} \tilde{\in}(Y, A)$.

Let $\tilde{\varepsilon}$ be such that $\tilde{l} \approx \widetilde{\varepsilon}$. Then for any $P_{\lambda}{ }^{x} \widetilde{\in}(Y, A)$, we have $\tilde{d}\left(P_{\lambda}{ }^{x}, P_{\mu}{ }^{y}\right) \widetilde{\Sigma} \tilde{l} \approx \tilde{\varepsilon}, \quad \forall P_{\mu}{ }^{y} \widetilde{\in}(Y, A)$.

$\Longrightarrow(Y, A) \widetilde{\subset} B\left(P_{\lambda}^{x}, \tilde{\varepsilon}\right)$.

Hence $(Y, A)$ is soft bounded.

Theorem 2.3 Finite union of soft bounded sets is soft bounded.

Proof: Let $\left(Y_{i}, A_{i}\right)$ be soft bounded sets for $1 \leq i \leq n$.

Then there exist $\widetilde{\varepsilon_{i}} \widetilde{\supset}$ and $P_{\lambda_{i}}{ }^{x_{i}} \widetilde{\in} \widetilde{X}$, such that

$\left(Y_{i}, A_{i}\right) \widetilde{\subset} B\left(P_{\lambda_{i}}^{x_{i}}, \widetilde{\varepsilon}_{i}\right), \quad \forall 1 \leq i \leq n$.

Let $(Y, A)=\bigcup_{i=1}^{n}\left(Y_{i}, A_{i}\right)$ and consider $P_{\lambda_{1}}^{x_{1}} \tilde{\in} \tilde{X}$.

Let $\tilde{\varepsilon}=\max \left\{\tilde{\varepsilon_{1}}, \widetilde{\varepsilon_{2}}, \ldots, \widetilde{\varepsilon_{n}}\right\}+\max \left\{\tilde{d}\left(P_{\lambda_{1}}{ }^{x_{1}}, P_{\lambda_{2}}{ }^{x_{2}}\right), \ldots \tilde{d}\left(P_{\lambda_{1}}{ }^{x_{1}}, P_{\lambda_{n}}{ }^{x_{n}}\right)\right\} \widetilde{0}$.

Now we show that $(Y, A) \widetilde{\subset} B\left(P_{\lambda_{i}}{ }^{x_{i}}, \tilde{\varepsilon}\right)$.

Let $P_{\mu}{ }^{y} \widetilde{\in}(Y, A)=\bigcup_{i=1}^{n}\left(Y_{i}, A_{i}\right)$. Then $P_{\mu}{ }^{y} \widetilde{\in}\left(Y_{j}, A_{j}\right)$ for some $\mathrm{j}$.

$\Rightarrow \tilde{d}\left(P_{\mu}{ }^{y}, P_{\lambda_{1}}{ }^{x_{1}}\right) \widetilde{d}\left(P_{\mu}{ }^{y}, P_{\lambda_{j}}{ }^{x_{j}}\right)+\tilde{d}\left(P_{\lambda_{j}}{ }^{x_{j}}, P_{\lambda_{1}}{ }^{x_{1}}\right)$

$$
\begin{aligned}
& \widetilde{\varepsilon_{j}}+\max \left\{\tilde{d}\left(P_{\lambda_{1}}{ }^{x_{1}}, P_{\lambda_{2}}{ }^{x_{2}}\right), \ldots \tilde{d}\left(P_{\lambda_{1}}{ }^{x_{1}}, P_{\lambda_{n}}{ }^{x_{n}}\right)\right\} \\
& \approx \max \left\{\widetilde{\varepsilon_{1}}, \widetilde{\varepsilon_{2}}, \ldots, \widetilde{\varepsilon_{n}}\right\}+\max \left\{\tilde{d}\left(P_{\lambda_{1}}{ }^{x_{1}}, P_{\lambda_{2}}{ }^{x_{2}}\right), \ldots \tilde{d}\left(P_{\lambda_{1}}{ }^{x_{1}}, P_{\lambda_{n}}{ }^{x_{n}}\right)\right\}=\tilde{\varepsilon}
\end{aligned}
$$

$\Rightarrow P_{\mu}{ }^{y} \widetilde{\in} B\left(P_{\lambda_{i}} x_{i}, \tilde{\varepsilon}\right)$

$\Rightarrow(Y, A) \simeq B\left(P_{\lambda_{i}}^{x_{i}}, \tilde{\varepsilon}\right)$. 
Remark 2.4: The above result is not true in general for arbitrary union.

For example, take $\tilde{X}=\widetilde{\mathbb{R}}$ and $\left(Y_{n}, A_{n}\right)=[-\tilde{n}, \tilde{n}]$.

Then each $\left(Y_{n}, A_{n}\right)$ is bounded as $\operatorname{diam}\left(\left(Y_{i}, A_{i}\right)\right) \simeq \tilde{2} \tilde{n}$.

But $\bigcup_{n=1}^{\infty}\left(Y_{n}, A_{n}\right)=\bigcup_{n=1}^{\infty}[-\tilde{n}, \tilde{n}]=\widetilde{\mathbb{R}}$, which is not bounded.

3. Soft Totally Bounded Sets

Definition 3.1 Let $(\tilde{X}, \tilde{d} E)$ be a soft metric space and $(Y, A) \widetilde{\subset}(\tilde{X}, E)$. We say that $(Y, A)$ is soft totally bounded if for a given $\tilde{\varepsilon} \widetilde{\mathcal{0}}$, there exist finitely many soft points $P_{\lambda_{i}} x_{i} \tilde{\in} \tilde{X}$ such that $(Y, A) \simeq \bigcup_{i=1}^{n} B\left(P_{\lambda_{i}}^{x_{i}}, \tilde{\varepsilon}\right)$.

Theorem 3.2 If $(Y, A)$ is soft totally bounded then for given $\widetilde{\varepsilon} \widetilde{\sim} \tilde{0}$, there exist $P_{\lambda_{i}}{ }^{x_{i}} \widetilde{\in}(Y, A)$ such that $(Y, A) \widetilde{\subset} \bigcup_{i=1}^{n} B\left(P_{\mu_{i}}{ }^{y i}, \tilde{\varepsilon}\right)$.

Proof:- Let $\tilde{\varepsilon} \Im \tilde{0}$. Since $(Y, A)$ is soft totally bounded, there exist $P_{\lambda_{i}}^{x_{i}} \widetilde{\in} \tilde{X}$ such that $(Y, A) \simeq \bigcup_{i=1}^{n} B\left(P_{\lambda_{i}} x_{i}, \frac{\tilde{\tilde{s}}}{2}\right)$.

Without loss of generality, we can assume that $(Y, A) \widetilde{\cap} B\left(P_{\lambda_{i}}^{x_{i}}, \frac{\tilde{\xi}}{2}\right) \neq \emptyset$ for all i.

So let $P_{\mu_{i}}{ }^{y i} \widetilde{\in}(Y, A) \widetilde{\cap} B\left(P_{\lambda_{i}}^{x_{i}}, \frac{\tilde{s}}{2}\right)$.

Now we show that $(Y, A) \widetilde{\subset} \bigcup_{i=1}^{n} B\left(P_{\mu_{i}}{ }^{y i}, \widetilde{\mathcal{\varepsilon}}\right)$

Let $P_{\gamma}{ }^{y} \widetilde{\in}(Y, A)$. Then there exists $j$ such that $P_{\gamma}{ }^{y} \widetilde{\in} B\left(P_{\lambda_{j}}{ }^{x_{j}}, \frac{\tilde{\tilde{s}}}{2}\right)$

$\Rightarrow \tilde{d}\left(P_{\gamma}{ }^{y}, P_{\mu_{j}}{ }^{y_{j}}\right) \widetilde{\widetilde{d}}\left(P_{\mu}{ }^{y}, P_{\lambda_{j}}{ }^{x_{j}}\right)+\tilde{d}\left(P_{\lambda_{j}}{ }^{x_{j}}, P_{\mu_{j}}{ }^{y_{j}}\right)$ $\approx \frac{\tilde{\varepsilon}}{2}+\frac{\tilde{\varepsilon}}{2}=\widetilde{\varepsilon}$

$\Rightarrow(Y, A) \widetilde{\subset} \bigcup_{i=1}^{n} B\left(P_{\mu_{i}} y_{i}, \widetilde{\varepsilon}\right)$

i.e $(Y, A)$ can be covered by finitely many $\widetilde{\mathcal{E}}$-balls, each centered at a point in $(Y, A)$.

Theorem 3.3:- $(Y, A)$ is soft totally bounded if and only if given $\tilde{\varepsilon}>\tilde{0}$ there exist finitely many soft soft subsets $\left(Y_{i}, A i\right) \widetilde{\subset}(Y, A)$ with $\operatorname{diam}\left(\left(Y_{i}, A i\right)\right) \stackrel{\gtrless}{\approx} \tilde{\varepsilon}_{\text {such that }}(Y, A) \widetilde{\subset} \bigcup_{i=1}^{n}\left(Y_{i}, A i\right)$. 
Proof:- Suppose that $(Y, A)$ is soft totally bounded and $\widetilde{\mathcal{E}} \widetilde{\sim} \tilde{0}$ be given.

Then by Theorem 3.2, there exist $P_{\lambda_{i}}{ }^{y i} \widetilde{\in}(Y, A)$ such that $(Y, A) \widetilde{\subset} \bigcup_{i=1}^{n} B\left(P_{\lambda_{i}}{ }^{y i}, \frac{\tilde{\xi}}{2}\right)$ and $(Y, A) \widetilde{\cap} B\left(P_{\lambda_{i}} y_{i}, \frac{\tilde{\xi}}{2}\right) \neq \emptyset$ for all $i$.

Let $\left(Y_{i}, A i\right)=(Y, A) \widetilde{\cap} B\left(P_{\lambda_{i}} y_{i}, \frac{\tilde{\tilde{\sigma}}}{2}\right)$

Then $\left(Y_{i}, A i\right) \widetilde{C}(Y, A)$ and $\operatorname{diam}\left(\left(Y_{i} ; A i\right)\right) \widetilde{2} \frac{2 \tilde{\varepsilon}}{2}=\tilde{\varepsilon}$.

Conversely, suppose for given $\tilde{\varepsilon} \Im \tilde{0}$ there are $\left(Y_{i} ; A i\right) \widetilde{\subset}(Y, A)$ such that

$(Y, A) \simeq \bigcup_{i=1}^{n}(Y ; A i)$ With $\operatorname{diam}\left(\left(Y_{i} ; A i\right)\right) \approx \frac{\tilde{\varepsilon}}{2}$. Then $\left(Y_{i}, A_{i}\right) \simeq B\left(P_{\lambda_{i}}{ }^{x_{i}}, \tilde{\varepsilon}\right)$

$\Rightarrow(Y, A) \simeq \bigcup_{i=1}^{n}\left(Y ; A_{i}\right) \simeq \bigcup_{i=1}^{n} B\left(P_{\lambda_{i}}^{x_{i}}, \tilde{\varepsilon}\right)$.

$\Rightarrow(Y, A)$ is soft totally bounded.

Theorem 3.4 In soft discrete metric space, a soft set is soft totally bounded if and only if it is finite.

Proof:- Let $(Y, A)$ be soft totally bounded.

Since $(Y, A)$ is soft totally bounded, for $\tilde{\varepsilon}=\tilde{1}$ there exist $P_{\lambda_{i}}{ }^{y_{i}} \tilde{\in}(Y, A)$ such that,

$(Y, A) \widetilde{\subset} \bigcup_{i=1}^{n} B\left(P_{\lambda_{i}}{ }^{y i}, \tilde{1}\right)$

But in discrete metric space, $B\left(P_{\lambda_{i}}{ }^{y i}, \tilde{1}\right)=\left\{P_{\lambda_{i}}{ }^{y_{i}}\right\}$. $\Rightarrow(Y, A) \simeq \bigcup_{i=1}^{n}\left\{P_{\lambda_{i}} y_{i}\right\}$

Hence $(Y, A)$ is finite.

Conversely, suppose $(Y, A)$ is finite, say $(Y, A)$ is the set of $P_{\lambda_{1}}^{y_{1}}, \ldots P_{\lambda_{n}}{ }^{y_{n}}$.

Let be $\tilde{\varepsilon}\left\ulcorner\right.$ o given. Then $(Y, A)=\bigcup_{i=1}^{n}\left\{P_{\lambda_{i}}{ }^{y}\right\} \widetilde{\subset} \bigcup_{i=1}^{n} B\left(P_{\lambda_{i}}{ }^{y_{i}}, \tilde{\varepsilon}\right)$

$\Rightarrow(Y, A)$ is soft totally bounded.

Theorem 3.5 A soft totally bounded set is soft bounded.

Proof:- Let $(Y, A)$ be a soft totally bounded. Then for $\tilde{\varepsilon}=\widetilde{1}$, there exist $P_{\lambda_{i}}{ }^{y i} \widetilde{\in}(Y, A)$ such that, $(Y, A) \widetilde{\subset} \bigcup_{i=1}^{n} B\left(P_{\lambda_{i}} y_{i}, \tilde{1}\right)$

Let $\tilde{\mathrm{r}}=\max \left\{\tilde{d}\left(P_{\lambda_{1}}{ }^{x_{1}}, P_{\lambda_{j}}{ }^{x_{j}}\right) / 2 \leq j \leq n\right\}+\tilde{1}$.

We now show that $(Y, A) \widetilde{\subset} B\left(P_{\lambda_{1}} y_{1}, \tilde{r}\right)$

Let $P_{\lambda}{ }^{y} \tilde{\in}(Y, A)$. Then there exists $j$ such that $P_{\lambda}{ }^{y} \widetilde{\in} B\left(P_{\lambda_{j}}{ }^{y j}, \tilde{1}\right)$. By triangle inequality,

$$
\begin{aligned}
& \tilde{d}\left(P_{\lambda}{ }^{y}, P_{\lambda_{1}}{ }^{y_{1}}\right) \approx \tilde{d}\left(P_{\lambda}{ }^{y}, P_{\lambda_{j}}{ }^{y j}\right)+\tilde{d}\left(P_{\lambda_{j}}{ }^{y_{j}}, P_{\lambda_{1}}{ }^{y_{1}}\right) \\
& \approx \tilde{1}+\max \left\{\tilde{d}\left(P_{\lambda_{1}}{ }^{x_{1}}, P_{\lambda_{j}}{ }^{x_{j}}\right) / 2 \leq j \leq n\right\}=\widetilde{r} \\
& \Rightarrow P_{\lambda}{ }^{y} \widetilde{\in} B\left(P_{\lambda_{1}}{ }^{y_{1}}, \tilde{r}\right) \\
& \Rightarrow(Y, A) \simeq B\left(P_{\lambda_{1}}{ }^{y_{1}}, \tilde{r}\right) .
\end{aligned}
$$

Hence $(Y, A)$ is soft bounded.

Remark:- Converse of the above theorem is not true in general. 
Example 3.6:- Let $(Y, A)=[0,1]$ be an infinite soft subset of a discrete metric space $(\tilde{R}, \tilde{d}, E)$. where $\tilde{d}\left(P_{\lambda}{ }^{y}, P_{\mu_{1}}{ }^{y}\right)=|\tilde{x}-\tilde{y}|+|\tilde{\lambda}-\tilde{\mu}|$ As, $(Y, A) \simeq B\left(P_{\lambda}{ }^{y}, 2 \tilde{\varepsilon}\right),(Y, A)$ is bounded.

But since $(Y, A)$ is infinite, by Theorem 3.4, it is not soft totally bounded.

Theorem 3.7 The closure of a soft totally bounded subset of a soft metric space is soft totally bounded.

Proof:- Let $(\tilde{X}, \tilde{d} E)$ be a soft metric space and $(Y, A)$ be soft totally bounded.

Let $\tilde{\varepsilon}>\tilde{0}$ be a soft real number. Then there exist $P_{\lambda_{1}}{ }^{x_{1}}, P_{\lambda_{2}}{ }^{x_{2}}, \ldots P_{\lambda_{n}}{ }^{x_{n}}$ in $(Y, A)$

such that, $(Y, A) \widetilde{\complement} \mathrm{U}_{i=1}^{n} B\left(P_{\lambda_{i}} x_{i}, \frac{\tilde{s}}{2}\right)$.

Let $P_{\lambda}^{x} \tilde{\epsilon} \overline{(Y, A)}$. Then $(Y, A) \widetilde{\cap} B\left(P_{\lambda}^{x}, \frac{\tilde{\varepsilon}}{2}\right) \neq \emptyset$.

Let $P_{\lambda}^{y} \tilde{\epsilon}(Y, A)$ and $P_{\lambda}^{y} \tilde{\epsilon} B\left(P_{\lambda}^{x}, \frac{\tilde{\varepsilon}}{2}\right)$.

Then there exists $j$ such that $P_{\mu}^{y} \widetilde{\in} B\left(P_{\lambda_{j}} x_{j}, \frac{\tilde{\sigma}}{2}\right)$

$\tilde{d}\left(P_{\lambda}{ }^{x}, P_{\lambda_{\mathrm{j}}}{ }^{x_{j}}\right) \widetilde{d}\left(P_{\lambda}{ }^{x}, P_{\mu}{ }^{\mathrm{y}}\right)+\tilde{d}\left(P_{\mu}{ }^{\mathrm{y}}, P_{\lambda_{\mathrm{j}}}{ }^{x_{j}}\right)$ $\approx \frac{\tilde{\varepsilon}}{2}+\frac{\tilde{\varepsilon}}{2}=\tilde{\varepsilon}$.

$\Rightarrow P_{\lambda}^{x} \widetilde{\in} B\left(P_{\lambda_{\mathrm{j}}}^{x_{\mathrm{j}}}, \tilde{\varepsilon}\right)$

$\Rightarrow(Y, A) \simeq B\left(P_{\lambda_{\mathrm{i}}}{ }^{{ }_{11}}, \tilde{\varepsilon}\right)$

Hence $(Y, A)$ is soft totally bounded.

Theorem 3.8 A subspace of a soft totally bounded metric space is soft totally bounded.

Proof:- Let $(\tilde{X}, \tilde{d} E)$ be a soft totally bounded metric space.

Let $\tilde{\varepsilon} \sim 0$ be a soft real number. Then there exist $P_{\lambda_{1}}{ }^{x_{1}}, P_{\lambda_{2}}{ }^{x_{2}}, \ldots P_{\lambda_{n}}{ }^{x_{n}}$ in $(X, E)$ such that $(X, E) \simeq \bigcup_{i=1}^{n} B\left(P_{\lambda_{i}} \mathrm{x}_{\mathrm{i}}, \tilde{\varepsilon}\right)$.

Let $(\mathrm{Y}, \mathrm{A})$ be a soft subspace of $(\tilde{X}, \tilde{d} E)$.

Define $B^{(Y, A)}\left(P_{\lambda_{i}}{ }^{x_{i}}, \tilde{\varepsilon}\right)=\left\{P_{\mu}{ }^{y} \widetilde{\in}(\mathrm{Y}, \mathrm{A}) \mid \tilde{d}\left(P_{\mu}{ }^{\mathrm{y}}, P_{\lambda_{\mathrm{i}}}{ }^{\mathrm{x}_{\mathrm{i}}}\right) \widetilde{\varepsilon}\right\}$

Now we show that $(Y, A) \simeq \bigcup_{i=1}^{n} B^{(Y, A)}\left(P_{\lambda_{i}}{ }^{x_{i}}, \tilde{\varepsilon}\right)$. 
Let $P_{\gamma}{ }^{z} \widetilde{\in}(\mathrm{Y}, \mathrm{A})$. Then $P_{\gamma}{ }^{z} \widetilde{\in}(X, E) \widetilde{\subset} \bigcup_{i=1}^{n} B\left(P_{\lambda_{i}}{ }^{\mathrm{x}_{\mathrm{i}}}, \tilde{\varepsilon}\right)$

So there exists $j$ such that $P_{\gamma}{ }^{z} \widetilde{\in} B\left(P_{\lambda_{j}}{ }^{x_{j}}, \tilde{\varepsilon}\right)$

$$
\begin{aligned}
& \Rightarrow \tilde{d}\left(P_{\lambda_{j}}{ }^{y_{j}}, P_{\gamma}{ }^{z}\right) \widetilde{\underline{\varepsilon}} \\
& \Rightarrow P_{\gamma}{ }^{z} \widetilde{\in} B^{(Y, A)}\left(P_{\lambda_{i}}{ }^{x_{i}}, \tilde{\varepsilon}\right) \\
& \Rightarrow(\mathrm{Y}, \mathrm{A}) \widetilde{\subset} \bigcup_{i=1}^{n} B^{(Y, A)}\left(P_{\lambda_{i}}{ }^{x_{i}}, \tilde{\varepsilon}\right)
\end{aligned}
$$

Therefore, $(Y, A)$ is soft totally bounded.

Theorem 3.9 Every soft totally bounded metric space is soft separable.

Proof:- Let $(\tilde{X}, \tilde{d} E)$ be a soft totally bounded metric space. Then for each $\tilde{\varepsilon} \sim \tilde{0}$, there exist $m$ such that $\frac{\widetilde{1}}{m} \approx \tilde{\varepsilon}$ and $\frac{\widetilde{1}}{m}-$ net $\left(\mathrm{Y}_{\mathrm{m}}, \mathrm{Am}\right)$ for $\tilde{X} . \tilde{X} \widetilde{\subset} \cup_{i=1}^{n} B\left(P_{\lambda_{i}}{ }^{\mathrm{x}_{\mathrm{i}}}, \frac{\widetilde{1}}{m}\right)$

Let $(Y, A)=\cup_{i=1}^{\infty}\left(Y_{n}, A n\right)$. Then clearly it is countable.

We will show that $(Y, A)$ is dense in $(X, E)$.

Let $P_{\lambda}{ }^{x} \widetilde{\in}(\mathrm{X}, \mathrm{E})$. Then there exists $j$ such that $P_{\lambda}{ }^{x} \widetilde{\in} B\left(P_{\lambda_{j}}{ }^{\mathrm{x}}, \frac{\widetilde{1}}{m}\right)$

$\Rightarrow \tilde{d}\left(P_{\lambda^{x}}, P_{\lambda_{j}}{ }^{x_{j}}\right) \widetilde{\underline{1}} \approx \widetilde{\varepsilon}$

$$
\Rightarrow P_{\lambda_{j}}{ }^{x_{j}} \tilde{\in} B\left(P_{\lambda}^{x}, \tilde{\varepsilon}\right) .
$$

Since $P_{\lambda_{j}}{ }^{x_{j}} \widetilde{\epsilon}\left(Y_{m}, A_{m}\right)$, we have $P_{\lambda_{j}}{ }^{x_{j}} \widetilde{\in}(Y, A)$

$$
\Rightarrow P_{\lambda}{ }^{x} \widetilde{\in}(Y, A) \widetilde{\cap} B\left(P_{\lambda}^{x}, \widetilde{\varepsilon}\right) \neq \emptyset .
$$

Therefore, $(Y, A)$ is dense in $(X, E)$.

\section{Conclusions}

In this paper, in section 2, we proved a soft set is soft bounded if and only if it has finite diameter. We also derived some properties of soft bounded sets. In section 3, we have discussed soft totally bounded sets and proved some necessary conditions of soft totally bounded sets. We then proved that soft totally bounded set is separable.

\section{Acknowledgements}

One of the authors is thankful to UGC for providing financial support thorough MAULANA AZAD NATIONAL FELLOWHIP. 


\section{References}

[1] F.Feng,C.X.Li,B.Davvaz and M.I.Ali, Soft sets combined with fuzzy sets and rough sets: a tentative approach, Soft Computing 14 (2010) 8999-9911.

[2] P.K.Maji, R.Biswas and A.R.roy, Soft set theory, Comput. Math.Appl.45(2003)555-562.

[3] D.Molodstove, Soft set theory first result, Comput,Math.Appl.37(1999)19-31.

[4] Sadi Bayrramov, cigdem Gunduz(Aras) and murat I.Yazar, Some notes on compact sets in Soft metric space, arxiv:1308.3390v1..

[5] M.shabbir and M.Naz, On Soft topological spaces, Comput.Math.Appl.61(2011) 1786-1799.

[6] Sujoy Das and S.K. Samanta, Soft metric, Annals of Fuzzy mathematics and information,6(1)(2013)77-94. vol. 176 (2010), 61-107.

[7] Sujoy Das and S.K. Samanta, Soft real set, Soft real numbers and their properties, J.Fuzzy Math.20(3)92012)551-576. 